\title{
Análisis en retrospectiva del uso de plataformas de aprendizaje virtual como estrategia para evitar la deserción de los estudiantes de nuevo ingreso en Facultades de Ingeniería
}

\section{Retrospective analysis of the use of virtual learning platforms as a strategy to avoid the desertion of new students entering Engineering Faculties}

CANTO-CANUL, Roberto Carlos $\dagger^{*}$, LÓPEZ-MARTÍNEZ, Jorge Luis, SALAZAR-UITZ, Ricardo Rubén y LEZAMA-ZARRAGA, Francisco Román

Universidad Autónoma de Campeche, Campus V, Predio s/n por Av. Humberto Lanz Cárdenas y Unidad Habitacional Ecológica Ambiental, Col. Ex-Hacienda Kalá, CP 24085, San Francisco de Campeche, Cam., México.

ID $1^{\text {er }}$ Autor: Roberto Carlos, Canto-Canul / ORC ID: 0000-0003-2420-043X, CVU CONACYT ID: 391401

ID $1^{\text {er }}$ Coautor: Jorge Luis, López-Martínez / ORC ID: 0000-0002-1434-3966

ID $2^{\text {do }}$ Coautor: Ricardo Rubén, Salazar-Uitz / ORC ID: 0000-0003-2307-737X, CVU CONACYT ID: 416277

ID $3^{\text {er }}$ Coautor: Francisco Román, Lezama-Zarraga / ORC ID: 0000-0003-3397-7881, Researcher ID Thomson: U-12292018, CVU CONACYT ID: 205493

DOI: $10.35429 / J T A E .2020 .11 .4 .26 .33$

Recibido: 30 de Abril, 2020; Aceptado 30 de Junio 2020

\begin{abstract}
Resumen
La gran mayoría de las escuelas y facultades de ingeniería en el mundo coinciden en tener altos índices de deserción durante el primer año de sus estudiantes de nuevo ingreso, originado principalmente por un bajo desempeño en las asignaturas de ciencias básicas. Algunas de estas instituciones académicas contrarrestan esta problemática con los llamados "semestre cero", con cursos propedéuticos presenciales o bien, con algún tipo de programa de tutoría o acompañamiento al estudiante de nuevo ingreso; pero ¿qué sucede cuando estas opciones no son posibles de implementar en la institución académica? En la Facultad de Ingeniería de la Universidad Autónoma de Campeche, se diseñó una estrategia de nivelación, mediante la implementación de un curso propedeútico virtual utilizando el contenido académico de la plataforma de aprendizaje en línea Khan Academy. Esta estrategia se ha aplicado en los últimos dos ciclos escolares (2018-2019 y 2019-2020) El presente proyecto consiste en el análisis en retrospectiva de estas dos generaciones y se hace una comparación con el ciclo escolar 2017-2018, en el cual no se había implementado esta estrategia
\end{abstract}

Plataformas de aprendizaje virtual, Innovación educativa, Matemáticas, Ciencias básicas

\begin{abstract}
Mostly of the engineering schools in the world coincide in having high dropout rates during the first year of their new students, mainly caused by low performance in basic science subjects. Some of these academic institutions counteract this problem with the so-called "zero semester", with face-to-face preparatory courses or, with some type of tutoring or accompaniment program for the new student; But what happens when these options are not possible to implement in the academic institution? In the Faculty of Engineering of the Autonomous University of Campeche, a leveling strategy was designed, through the implementation of a virtual propedeutic course using the academic content of the online learning platform Khan Academy. This strategy has been applied in the last two school years (2018-2019 and 20192020) This project consists of a retrospective analysis of these two generations and a comparison is made with the 2017 2018 school year, in which this strategy had not been implemented.
\end{abstract}

Virtual learning platforms, Educational innovation, Mathematics, Basic Sciences

Citación: CANTO-CANUL, Roberto Carlos, LÓPEZ-MARTÍNEZ, Jorge Luis, SALAZAR-UITZ, Ricardo Rubén y LEZAMA-ZARRAGA, Francisco Román. Análisis en retrospectiva del uso de plataformas de aprendizaje virtual como estrategia para evitar la deserción de los estudiantes de nuevo ingreso en Facultades de Ingeniería. Revista de Tecnología y Educación. 2020. 4-11: 26-33

\footnotetext{
* Correspondencia del Autor (Correo electrónico: roccanto@uacam.mx)

$\dagger$ Investigador contribuyendo como primer autor.
} 


\section{Introducción}

El bajo rendimiento académico es un problema generalizado al que se enfrentan los docentes del área de las matemáticas que imparten clases en los primeros semestres de la educación superior. Esta situación se presenta no solo a nivel nacional sino también a nivel internacional y es abordado por diversos autores (Gómez, 2011).

En este sentido, la innovación de los escenarios en los que se desarrollan los procesos de enseñanza-aprendizaje en el nivel superior son resultado de la transformación de las universidades que transitan de la práctica docente presencial a la virtual. Lo anterior replantea a la didáctica con nuevos procesos que incorporan metodologías flexibles y enfocadas en el aprendizaje del estudiante (Salinas y Marín, 2017).

El uso de plataformas digitales para aprender se ha vuelto más concurrido entre alumnos de nivel superior ya que regularmente el uso de estas plataformas, así como de la tecnología disponible y sus posibilidades educativas, especialmente haciendo referencia al aprendizaje ubicuo, en el cual cada persona puede aprender en cualquier lugar y momento, hacen que las fronteras entre lo formal y lo informal resulten cada vez menos nítidas y se entrecrucen (Salinas y Marín, 2017).

Los resultados del Plan Nacional para la Evaluación de Aprendizajes (PLANEA) implementado por la Secretaria de Educación Pública (SEP) a nivel nacional son importantes y tienen un alto impacto con los alumnos de nuevo ingreso a las licenciaturas de ingeniería del país y por su puesto con los alumnos en la facultad de ingeniería ya que vienen con conocimientos divergentes de sus diferentes instituciones de nivel medio superior en el área de matemáticas.

Los resultados de este plan en el año 2017, en el campo formativo de Matemáticas, se tiene que 6 de cada 10 estudiantes se ubica en el nivel I (66\%); casi 2 de cada 10 se ubican en el nivel II (23\%); en el nivel III, sólo 8 de cada 100 estudiantes (8\%) y en el nivel IV, casi 3 estudiantes de cada 100 (2.5\%), (SEP \& INEE, 2017).

Estos criterios bajos los cuales se obtuvieron estos resultados del programa PLANEA se presentan en la

\section{Tabla 9.}

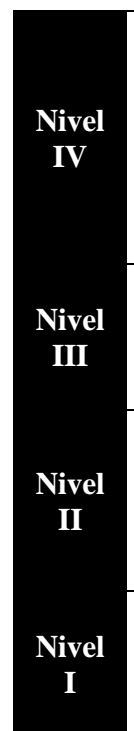

Dominan las reglas para transformar y operar con el
lenguaje matemático (por ejemplo, las leyes de los
signos); expresan en lenguaje matemático las
relaciones que existen entre dos variables de una
situación o fenómeno; y determinan algunas de sus
características (por ejemplo, deducen la ecuación de
la línea recta a partir de su gráfica).
Emplean el lenguaje matemático para resolver
problemas que requieren del cálculo de valores
desconocidos, y para analizar situaciones de
proporcionalidad.
Expresan en lenguaje matemático situaciones donde
se desconoce un valor o las relaciones de
proporcionalidad entre dos variables, y resuelven
problemas que implican proporciones entre
cantidades (por ejemplo, el cálculo de porcentajes).
Tienen dificultades para realizar operaciones con
fracciones y operaciones que combinen incógnitas o
variables (representadas con letras), así como para
establecer y analizar relaciones entre dos variables

Tabla 9 Nivel de logro del campo formativo de Matemáticas, SEP et al., (2017)

La Universidad Autónoma de Campeche (UACAM) en su Facultad de Ingeniería, ofrece seis licenciaturas cuyos primeros semestres se conforman principalmente por materias de ciencia básicas o de tronco común; las cuales son esenciales para la formación de un ingeniero ya que permiten el incremento de habilidades cognitivas para el desarrollo práctico y teórico de problemas.

La iniciativa parte de la imposibilidad de llevar a cabo un curso propedéutico presencial o la implementación de un semestre cero, debido principalmente a aspectos administrativos $y$ jurídicos de la propia Universidad.

Khan Academy es una de las plataformas de aprendizaje en línea más importantes en la actualidad, cuenta con más de 4.300 vídeos dirigidos a estudiantes de todos los niveles en las áreas de matemáticas y ciencias básicas. Además de vídeos instructivos, también ofrece ejercicios de práctica y un panel de aprendizaje personalizado; ha sido traducido a cerca de 40 idiomas y tiene presencia en todo el mundo.

Es importante mencionar que hay una gran variedad de plataformas de aprendizaje en línea, pero además Khan Academy cuenta con el respaldo de una de las empresas más importantes en México, la cual es propietaria de gran parte de la infraestructura en telecomunicaciones del país, gracias a este respaldo, se puede acceder al contenido de Khan Academy sin costo desde cualquier dispositivo móvil con acceso a la red telefónica. Este fue un factor decisivo para elegir 
esta plataforma, en beneficio de los estudiantes sin importar su situación socioeconómica.

\section{Metodología a desarrollar}

En el trabajo "Estrategia para mejorar el desempeño académico de los estudiantes de nuevo ingreso en Facultades de Ingeniería mediante el uso de plataformas de aprendizaje virtuales", (Canto R, Lezama F, Salazar R, 2019) presentado en al CIERMMI 2019, se describió a detalle la estructura del curso propedéutico y se analizó su implementación en el ciclo escolar 2018-2019. El punto de comparación del trabajo mencionado se hizo tomando en cuenta los resultados del examen diagnóstico y examen final, que se aplicaron al principio y final del curso propedéutico, respectivamente. En este análisis se pudo ver que hubo un incremento del $12 \%$ en el desempeño académico de los estudiantes que tomaron el curso. De igual forma se hizo un análisis de las calificaciones obtenidas, clasificándolas de acuerdo con su bachillerato de origen, esto con el fin de detectar un patrón de riesgo y atender a los alumnos oportunamente desde el momento en que llegan la Facultad.

En esta nueva etapa del proyecto, ya se tienen los resultados de una segunda aplicación del curso propedéutico correspondiente a la primera fase del ciclo 2019-2020. Estos datos recabados en los ciclos escolares mencionados, se compararán con el aprovechamiento escolar de los estudiantes de nuevo ingreso de la generación 2017-2018, cabe mencionar que, en este ciclo, aún no se había implementado este curso; lo cual hace relevante el análisis presentado, ya que los resultados serán un indicio en la importancia de aplicar estas estrategias para incrementar el desempeño académico de los estudiantes de nuevo ingreso y de la misma forma, reducir los índices de deserción en las Universidades.

Es importante mencionar que, con el fin de homogeneizar los resultados de las aplicaciones en diferentes ciclos escolares, el contenido del curso fue exactamente el mismo; de igual forma los exámenes de diagnóstico y final, consistieron en problemas de la misma complejidad. Cabe mencionar que el contenido del curso propedéutico, así como los exámenes aplicados fueron aprobados por la Academia de Ciencias Básicas y Matemáticas de la Facultad de Ingeniería para garantizar que sean pertinentes a los planes de estudio de los programas académicos.
En la Tabla 2 se presenta el número de estudiantes que ingresaron a la Facultad de Ingeniería según el programa educativo. Cabe mencionar que de Ingeniería Civil y Administración (ICA), se ofertan tres grupos por ciclo escolar; en el caso de Ingeniería en Mecatrónica (IM) e Ingeniería en sistemas computacionales (ISC) se ofertan dos grupos; y solo un grupo para el caso de Ingeniería en Tecnología de Software (ITS), Ingeniería en Mecánica Eléctrica (IME) e Ingeniería en Energía.

\begin{tabular}{|l|r|r|r|}
\cline { 2 - 3 } \multicolumn{1}{c|}{} & $\begin{array}{c}\text { Ciclo escolar 2017- } \\
\text { 2018 }\end{array}$ & \multicolumn{1}{c}{$\begin{array}{c}\text { Ciclo } \\
\text { escolar } \\
\text { 2018-2019 }\end{array}$} & $\begin{array}{c}\text { Ciclo } \\
\text { escolar } \\
\text { 2019-2020 }\end{array}$ \\
\hline ICA & 80 & 83 & 78 \\
\hline IM & 48 & 49 & 50 \\
\hline ISC & 51 & 46 & 55 \\
\hline ITS & 17 & 35 & 22 \\
\hline IME & 25 & 29 & 28 \\
\hline IE & 21 & 25 & 24 \\
\hline Total & $\mathbf{2 4 2}$ & $\mathbf{2 6 7}$ & $\mathbf{2 5 7}$ \\
\hline
\end{tabular}

Tabla 2 Número de alumnos por carrera por ciclo escolares

Fuente: Elaboración Propia

Los datos en la Tabla 2 corresponden al número de alumnos de nuevo ingreso en cada uno de los ciclos escolares que forman parte de este estudio; también se específica el programa educativo al cual pertenecen. Finalmente, se especifica con diferente color los ciclos escolares que participaron en el curso propedéutico virtual (color amarillo) de aquél que no contó con el curso propedéutico (color verde).

El curso propedéutico virtual consta de 182 actividades, las cuales fueron previstas a realizarse en un tiempo promedio de 35 a 40 horas dependiendo de la facilidad del estudiante a comprender los temas de manera autodidacta por medio de la plataforma. El curso propedéutico está conformado de cuatro temas principales:

\section{Pre-álgebra. \\ 2. Fundamentos de álgebra. \\ 3. Álgebra 2. \\ 4. Trigonometría.}

La distribución de actividades clasificada por el tipo de recurso y por el tema al que pertenece, se presenta en la Tabla 3 


\begin{tabular}{|l|r|r|r|r|}
\multicolumn{1}{c|}{} & \multicolumn{1}{c|}{\begin{tabular}{c} 
Pre \\
\multicolumn{1}{l|}{ álgebra }
\end{tabular}} & \multicolumn{1}{c|}{\begin{tabular}{c} 
Fund. \\
de \\
\multicolumn{1}{c|}{ Álgebra }
\end{tabular}} & \multicolumn{1}{c|}{$\begin{array}{c}\text { Algebra } \\
\mathbf{2}\end{array}$} & \multicolumn{2}{c|}{$\begin{array}{c}\text { Trigono- } \\
\text { metría }\end{array}$} \\
\hline Videos & 13 & 35 & 19 & 9 \\
\hline Ejercicios & 16 & 27 & 15 & 7 \\
\hline Cuestionario & 3 & 5 & 5 & 0 \\
\hline Pruebas & 0 & 3 & 0 & 1 \\
\hline Artículos & 6 & 6 & 7 & $\mathbf{2 2}$ \\
\hline Subtotal & $\mathbf{3 8}$ & $\mathbf{7 6}$ & $\mathbf{4 6}$ & \\
\hline
\end{tabular}

Tabla 3 Estructura del curso propedéutico Fuente: Elaboración Propia

Antes de realizar el curso propedéutico se aplicó un examen diagnóstico, el cual contó con 10 problemas de dificultad media, para medir el nivel de conocimiento de los estudiantes de nuevo ingreso. Posterior al curso, se aplicó una evaluación final con la misma dificultad, así como el número de preguntas.

Es importante mencionar que ambas evaluaciones se aplicaron por medio de la plataforma Google Formularios, esto con el objetivo de mantener la tendencia del uso de herramientas virtuales y tecnologías de información.

\section{Comparación de las dos primeras aplicaciones del curso propedéutico virtual}

Primeramente, se presentarán los resultados individuales de cada una de las aplicaciones del curso propedéutico:

En el ciclo escolar 2018-2019 se inscribieron 267 estudiantes en las seis diferentes carreras. Los resultados de su examen diagnostico (justo antes del curso propedéutico) tuvo un promedio grupal de 56.68 puntos, en la Gráfica 1 se puede ver la distribución normal de estos resultados.

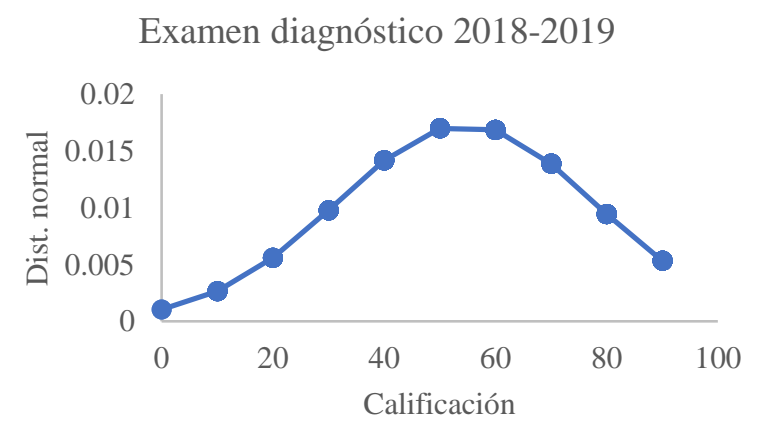

Grafica 1 Resultados del examen diagnóstico 2018-2019 Fuente: Elaboración Propia
Haciendo referencia al examen final, el cual se aplica inmediatamente después de terminar el curso propedéutico, se obtuvo una media de 67.81 puntos, lo cual nos indica que se presentó un incremento de 11.13 puntos en el nivel de aprovechamiento general en el área de ciencias básicas y matemáticas. La distribución normal de estos resultados se puede ver en la Gráfica 2.

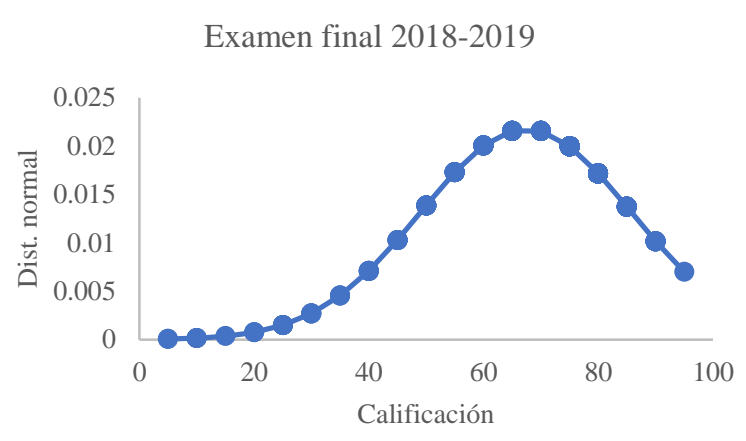

Grafica 2 Resultados del examen final 2018-2019 Fuente: Elaboración Propia

En cuanto a la segunda aplicación del curso propedéutico, llevada a cabo en el ciclo escolar 2019-2020 se inscribieron 257 estudiantes en las seis diferentes carreras. Los resultados de su examen diagnostico (justo antes del curso propedéutico) tuvo un promedio grupal de 42.41 puntos, en la Gráfica 3 se puede ver la distribución normal de estos resultados.

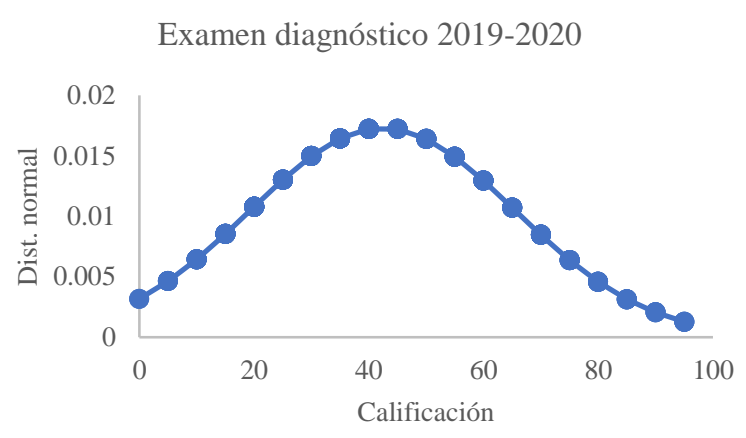

Grafica 3 Resultados del examen diagnóstico 2019-2020 Fuente: Elaboración Propia

Del examen final de esta etapa, se puede resaltar que la media fue de 58.89 puntos, lo cual nos indica que se presentó un incremento de 16.48 puntos en el nivel de aprovechamiento general en el área de ciencias básicas y matemáticas. La distribución normal de estos resultados se puede ver en la Gráfica 4 


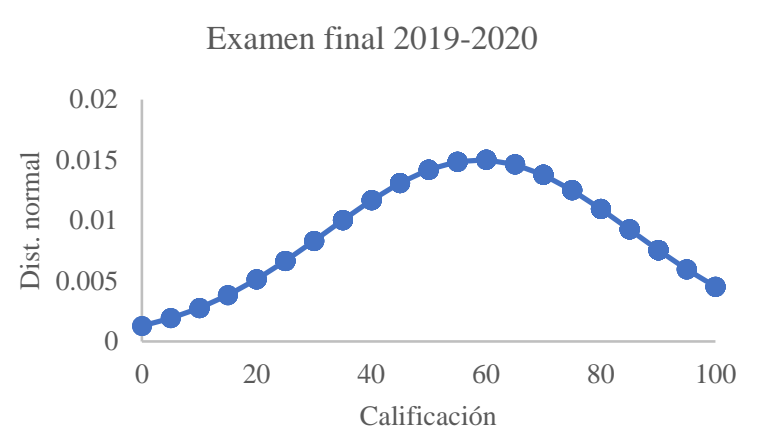

Grafica 4 Resultados del examen final 2019 Fuente: Elaboración Propia

Es muy importante mencionar que estos resultados indican que sí existe un incremento en el desempeño académico de los estudiantes al pasar por el proceso del curso propedéutico; es decir, al hacer estas mediciones únicamente con exámenes de entrada y salida, se entiende que el curso tiene un impacto inmediato (corto plazo), pero no justifica del todo que tenga resultados a mediano o largo plazo.

\section{Resultados}

Para la segunda parte de los resultados, se tomará en consideración el promedio de las asignaturas al finalizar el primer semestre del respectivo ciclo escolar. Debido a que el curso propedéutico consta de cuatro pilares principales: Pre-álgebra, Fundamentos de álgebra, Álgebra 2 y trigonometría; únicamente se tomarán las materias cuyos planes de estudio incluyan temas afines al curso propedéutico o cuyo aprovechamiento dependa de ellos.

En la Tabla 3 se muestran las asignaturas de ciencias básicas que se imparten en las diferentes carreras de la facultad. Las siglas utilizadas para las asignaturas corresponden a: Geometría analítica (GA), Álgebra y geometría analítica (AGA), Álgebra superior (AS), Cálculo diferencial (CD) y Cálculo diferencial e integral (CDI), respectivamente.

\begin{tabular}{|l|c|c|c|c|c|}
\multicolumn{1}{c}{} & \multicolumn{1}{c}{ GA } & AGA & AS & CD & CDI \\
\hline ISC & $\mathrm{X}$ & & $\mathrm{X}$ & $\mathrm{X}$ & \\
\hline IE & $\mathrm{X}$ & & $\mathrm{X}$ & $\mathrm{X}$ & \\
\hline ICA & & $\mathrm{X}$ & & & $\mathrm{X}$ \\
\hline IM & & $\mathrm{X}$ & & & $\mathrm{X}$ \\
\hline ITS & & $\mathrm{X}$ & & & \\
\hline IME & & $\mathrm{X}$ & & & \\
\hline
\end{tabular}

Tabla 4 Asignaturas de ciencias básicas en las carreras de la facultad de ingeniería UACAM

Fuente: Elaboración Propia
Estas asignaturas se compararán de manera grupal y por generación para obtener un panorama general del impacto del curso propedéutico en la facultad de Ingeniería de la UACAM. Hay que recordar que en el ciclo escolar 2017-2018 no se contaba con curso propedéutico.

En las tablas 5, 6 y 7 se puede ver el promedio por generación de cada una de las asignaturas de ciencias básicas, es importante mencionar que para el caso en que hay más de un grupo por asignatura, el resultado presentado es el promedio de ellos. En cada uno de los programas académicos se resaltó el promedio más alto.

Analizando la Tabla 5, específicamente el caso de Ingeniería en Sistemas Computacionales se puede ver que en la asignatura de geometría analítica la calificación promedio antes del curso propedéutico era $6.2 \mathrm{y}$ luego de la implementación del curso, subió a 6.8 y 7.2 en los ciclos posteriores. En el caso de Álgebra superior, hubo un decremento de $6.5 \mathrm{a}$ 6.1 luego del curso propedéutico, sin embargo, en la siguiente aplicación se mantiene al promedio inicial de 6.5. Si se analiza el caso de Ingeniería en Energía, se conserva esta tendencia de que los promedios son superiores luego del curso propedéutico, esto ocurre en todas las asignaturas que se evaluaron.

\begin{tabular}{|c|c|c|c|c|}
\hline & $\begin{array}{l}\frac{1}{0} \\
\frac{5}{8} \\
8 \\
0 \\
0 \\
0 \\
0\end{array}$ & 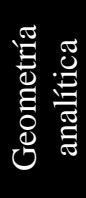 & 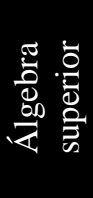 & 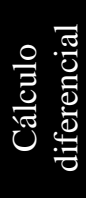 \\
\hline \multirow{3}{*}{ ISC } & $17-18$ & 6.20 & 6.5 & 6.2 \\
\hline & 18-19 & 6.8 & 6.1 & 7.1 \\
\hline & 19-20 & 7.2 & 6.5 & 6.9 \\
\hline & & & & \\
\hline \multirow{3}{*}{ IE } & 17-18 & 6.0 & 7.5 & 5.2 \\
\hline & 18-19 & 7.5 & 7.6 & 5.9 \\
\hline & $19-20$ & 7.1 & 7.6 & 6.1 \\
\hline
\end{tabular}

Tabla 5 Promedios grupal en asignaturas de ciencias básicas por ciclo escolar de la carrera de ISC e IE Fuente: Elaboración Propia

En la Tabla 6 se ven los resultados de Ingeniería civil y administración en la que podemos ver un decremento en el resultado de 'Algebra y geometría analítica luego de la primera aplicación del curso propedéutico, sin embargo, se presenta un incremento notorio en la segunda aplicación. 
En el caso de Cálculo diferencia e integral, se mantuvo el promedio luego de la primera aplicación, pero incrementa levemente luego de la segunda aplicación. Caso similar ocurre si se analiza la carrera de ingeniería en Mecatrónica.

\begin{tabular}{|c|c|c|c|}
\hline & 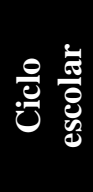 & 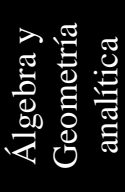 & 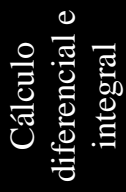 \\
\hline \multirow{3}{*}{ ICA } & $17-18$ & 7.1 & 6.5 \\
\hline & 18-19 & 6.8 & 6.5 \\
\hline & 19-20 & 8.1 & 7.1 \\
\hline \multirow{3}{*}{ IM } & $17-18$ & 72 & 6.8 \\
\hline & $18-19$ & 7.8 & 7.6 \\
\hline & $19-20$ & 6.9 & 7.2 \\
\hline
\end{tabular}

Tabla 6 Promedio grupal en asignaturas de ciencias básicas por ciclo escolar de la carrera de ICA e IM Fuente: Elaboración Propia

Salvo unas excepciones, se puede ver que los promedios tienen a subir a partir del ciclo escolar 18-19, lo que indica que el curso propuesto está teniendo un impacto positivo en el desempeño académico de la comunidad estudiantil, esto se puede ver si se analiza cada caso particular en las tablas presentadas.

\begin{tabular}{|c|c|c|}
\multicolumn{1}{c}{} & \multicolumn{1}{c|}{ Ciclo escolar } & $\begin{array}{c}\text { Álgebra y Geometría } \\
\text { analítica }\end{array}$ \\
\hline \multirow{3}{*}{ ITS } & $17-18$ & 5.4 \\
\cline { 2 - 3 } & $18-19$ & 6.8 \\
\cline { 2 - 3 } & $19-20$ & 6.8 \\
\hline \multirow{3}{*}{ IME } & $17-18$ & 7.5 \\
\cline { 2 - 3 } & $18-19$ & 6.9 \\
\cline { 2 - 3 } & $19-20$ & 7.5 \\
\hline
\end{tabular}

Tabla 7 Promedio grupal en asignaturas de ciencias básicas por ciclo escolar de la carrera de ITS e IME Fuente: Elaboración Propia

\section{Agradecimientos}

Se agradece a la Universidad Autónoma de Campeche y a la Facultad de Ingeniería las facilidades y el apoyo otorgados para poder desarrollar e implementar este proyecto de investigación.

\section{Conclusiones}

El presente trabajo es una recopilación del trabajo realizado durante los últimos tres ciclos escolares en la Facultad de Ingeniería de la Universidad Autónoma de Campeche.
La población para estudiar estuvo conformada por tres generaciones consecutivas durante el primer año de su ingreso a la facultad.

En los ciclos escolares 2018-2019 y 2019-2020 se implementó respectivamente, un programa de curso propedéutico virtual, el cual se conformó en su totalidad con el contenido disponible en la plataforma Khan Academy. Con la aprobación de la Academia de Ciencias Básicas y Matemáticas de la Facultad de Ingeniería se propuso un curso que constó de 182 actividades conformados por videos, lecturas y ejercicios prácticos elegidos cuidadosamente para el desarrollo de habilidades cognitivas necesarias durante el primer año de la carrera.

Analizando los cursos propedéuticos de manera individual, los resultados presentados muestran una tendencia positiva en el desempeño de los estudiantes al haber incrementado el promedio general y haber movido la distribución normal hacia valores mayores de desempeño. Analizando los cursos propedéuticos de manera conjunta, se hizo un seguimiento académico muy minucioso a cada uno de los grupos que participaron en el proceso experimental, ya que el desempeño de ellos se tomaría como base para calcular las estadísticas del aprovechamiento e impacto del curso propedéutico en la comunidad estudiantil.

Los resultados obtenidos muestran que el curso propedéutico ha tenido un impacto positivo en los promedios grupales ya que, en su mayoría, se han incrementado en diferentes porcentajes, en menos casos se ha mantenido igual y en una minoría de ellos el promedio ha descendido. Es importante resaltar que hay factores que no se están tomando en cuenta pero que pudieran intervenir indirectamente en estos resultados; en términos generales, el panorama del estudio presentado es favorable y cumple en alguna medida con los objetivos planteados inicialmente con el proyecto.

En el caso de la deserción, también se han visto resultados positivos, ya que en términos generales se ha reducido se redujo en $8 \%$ la deserción si se comparan los ciclos escolares 2017-2018 con el ciclo 2018-2019; y cerca del $10 \%$ si se compara el ciclo 17-18 con el ciclo escolar 2019-2020. 
Hablando en términos de reprobación, también ha habido un decremento en los números que se reportaban hasta finales del 2017, toda vez, que en la legislación universitaria se estipula que, en el primer semestre de cualquier carrera, el estudiante debe aprobar por lo menos el $50 \%$ de sus materias para poder continuar al siguiente, de lo contrario, causaría baja, sin opción a continuar sus estudios en el programa educativo que está cursando.

Se pretende implementar con el tiempo este proyecto de manera institucional y hacerlo extensivo a las preparatorias y todas aquellas facultades de la Universidad que requieran nivelar a sus estudiantes en temas de ciencias básicas.

\section{Referencias}

Canto R., Salazar, R., Lezama, F. (2019) Estrategia para mejorar el desempeño académico de los estudiantes de nuevo ingreso en Facultades de Ingeniería mediante el uso de plataformas de aprendizaje virtuales. Revista de Ciencias de la educación. Vol.3 No.10 ECORFAN

Domínguez, I. H., Cuenca, R. T., \& Hernández, A. D. M. (2019). Innovación docente basada en el empleo de objetos virtuales de aprendizaje de Lengua Española (Original). Roca. Revista científico-educacional de la provincia Granma, 14(5), 50-64.

Gómez, O. A. (2011). Ruta de apoyo pedagógico para la enseñanza de geometría y trigonometría, en el curso 'matemáticas básicas' de la Universidad Nacional de Colombia Sede Medellín. (tesis doctoral). Universidad Nacional de Colombia, Medellín.

Hernández Reyes, G. \& Azueta León, C. (2018). Impulsando la educación en el aula por medio de los recursos tecnológicos libres de gamificación. Memorias del Congreso Internacional de Investigación Academia Journals Chetumal 2018, V 10, pp 2795-2800, http://www.academiajournals.com/pub-chetumal.

Jiménez Jiménez, L. (2020). Nuevas tecnologías aplicadas a la enseñanza del análisis en secundaria: Introducción al concepto de optimización.
Muñoz Amariles, M. \& Álvarez González, J. (2015). Ingeniería Mecánica: las Matemáticas en los últimos 33 años y visión actual de este campo. Memorias del XXI Congreso Internacional Anual de la SOMIM 2015 Coatzacoalcos, Veracruz, México, pp 414-414, http://somim.org.mx/memorias-somim/

Perera, Díaz \& Salinas Padilla, Heidi Angélica. (2019). Curso de nivelación algebraica para incrementar el rendimiento académico en estudiantes de ingeniería en un ambiente virtual de aprendizaje. 8. 34.

Salinas, J., y Marín, J. (2017). La universidad entre lo real y lo virtual: una trayectoria no lineal para la didáctica universitaria. Notandum, 44-45. Recuperado de http://www.hottopos.com/notand44/1SalinasMa rin.pdf.

Torres, S., \& Elena, R. (2019). Aplicación del modelo BLENDED LEARNING para mejorar el rendimiento académico de los estudiantes de la asignatura de dinámica de sistemas de la escuela profesional de ingeniería de sistemas e informática de la Universidad Nacional de San Martín-Tarapoto.

Ramírez Ochoa, M. \& Vizcarra Brito, J. (2016). Desarrollo De Habilidades Matemáticas En Estudiantes Normalistas Mediante Khan Academy. Ra Ximhai [en línea] 2016, 12 (Juliodiciembre): [Fecha de consulta: 12 de julio de 2019] Disponible en: http://www.redalyc.org/articulo.oa?id=4614819401 9, ISSN 1665-0441

SEP \& INEE (2015). Difusión de resultados de PLANEA Media superior 2015. Publicación de Resultados Primera Aplicación 2015. México: PLANEA. Recuperado de: http://planea.sep.gob.mx/content/general/docs/2015/ PLANEA_MS2015_publicacion_resultados_04081 5.pdf

SEP \& INEE (2017). Difusión de resultados de PLANEA Media superior 2017. PLANEA Resultados Nacionales 2017, Educación Media Superior, Lenguaje y Comunicación/Matemáticas. México: PLANEA. Recuperado de: http://planea.sep.gob.mx/content/general/docs/2017/ ResultadosNacionalesPlaneaMS2017.PDF 
Roig-Vila, R., \& Moreno-Isac, V. (2020). El pensamiento computacional en Educación. Análisis bibliométrico y temático. Revista de Educación a Distancia, 20(63). 